Konsens gesucht

\title{
AV-Block bei Borreliose: Wie und wie lange therapis
}

Frage: Ein 47-jähriger Patient ohne Vorerkrankungen erleidet einen AV-Block Grad III und wird mit einem Schrittmacher versorgt. Eindeutige positive Borrelienserologie erreicht mich nach Entlassung aus dem Krankenhaus. Der Patient bekommt seit heute Ceftriaxon $2 \mathrm{~g}$ pro Tag. Die Dauer der intravenösen Therapie wird dennoch unterschiedlich angegeben und es wird teilweise mit Doxycyclin nachbehandelt. Gibt es unterdessen einen Konsens?

\section{Viele Steine, keine Pein Trotzdem Gallenblase rausnehmen?}

Frage: Es würde mich interessieren, ob nach dem heutigem Stand der Forschung eine absolute Indikation zur Cholezystektomie gegeben ist, wenn bei Vorliegen von multiplen Konkrementen in der Gallenblase sonographisch keine Gallenblasenflüssigkeit mehr nachweisbar ist. Ein asymptomatischer Patient sei vorausgesetzt. Bisher konnte ich in der Fachliteratur diesbezüglich keine Antwort finden.

Dr. Martin Held, München: Nach wie vor besteht eine absolute Indikation zur Cholezystektomie nur bei symptomatischer Cholezystolithiasis und Porzellangallenblase. In solchen Fällen besteht nämlich das Risiko einer malignen Transformation. Die von ihnen geschildete Situation stellt dementsprechend keine absolute Indikation zur Cholezystektomie dar.

Expertenrat Gastroenterologie www.springermedizin.de
Dr. Charalampos Kriatselis, Berlin:

Bezüglich der Therapie der Borreliose gibt es Empfehlungen von verschiedenen Gesellschaften, wie z. B. dem Center for Disease Control and Prevention, dem National Institute of Allergy and Infectious Disease (USA) und dem Robert Koch Institut. Hier wird die kardiale Beteiligung als Indikation für eine i.v.-Therapie mit Cephalosporinen der dritten Generation (z. B. Ceftriaxon $2 \mathrm{~g}$ täglich bei Erwachsenen) angeführt.
Die empfohlene Dauer dieser Behandlung beträgt 10 bis 28 Tage (meistens 14 Tage). Das Center for Disease Control and Prevention empfiehlt eine anschließende orale antibiotische Behandlung mit Doxycyclin (100 mg zweimal täglich) oder Amoxicillin (500 mg dreimal täglich) bzw. Cefuroxim-Axetil (500 mg zweimal täglich) für 14 Tage.

Expertenrat Rhythmusstörungen www.springermedizin.de

\section{Durch Pädiater verunsichert Doch besser keine Milch für Kinder?}

Frage: Ich empfehle bei Kleinkindern und Jugendlichen Milch als Calciumund Vitamin D-Zufuhr. Jedoch wird von Kinderärzten die Einnahme von Milch nicht angeraten. Warum?

Prof. Dr. Jürgen C. Frölich, Hannover: Bei der Empfehlung von Milch für Kinder ist das Alter des Kindes zu beachten. Die Ernährungskommission der Deutschen Gesellschaft für Kinderheilkunde und Jugendmedizin empfiehlt für die Ernährung von Kindern im ersten Lebensjahr, Kuhmilch und Kuhmilchprodukte nur in begrenztem Umfang und in denaturierter (erhitzter) Form (z. B. als Getreide-Milch-Brei) einzusetzen. Trinkmilch hat einen niedrigen Eisengehalt, behindert die Resorption von Nichthämeisen und kann bei Säuglingen okkulten Blutverlust im Stuhl hervorrufen (Stellungnahme abrufbar über www.fke-do.de). Für die optimale Entwicklung von Knochen und Zähnen bei Kindern ist eine Versorgung mit Calcium und Vitamin D wichtig. Milch- und Milchprodukte sind die beste Calciumquelle. Im Alter von vier bis sechs Jahren sollten Kinder $350 \mathrm{ml} \mathrm{Milch/-produkte} \mathrm{pro} \mathrm{Tag} \mathrm{zu}$ sich nehmen. Fettarme Varianten sind dabei $\mathrm{zu}$ bevorzugen. $\mathrm{Zu}$ beachten ist, dass „Kindermilchprodukte“ und Milchdesserts häufig viel Zucker und Fett enthalten. Calciumreiche Gemüsearten sind Brokkoli, Grünkohl, Lauch und Fenchel. Für die körpereigene Vitamin-D-Bildung ist viel Bewegung im Freien förderlich.

Expertenrat Pharmazie www.springermedizin.de 